\title{
Human body exposure to fixed potentials surfaces in power substations
}

\author{
C. González ${ }^{1}$, A. Peratta ${ }^{1} \&$ D. Poljak ${ }^{2}$ \\ ${ }^{1}$ Wessex Institute of Technology, UK \\ ${ }^{2}$ University of Split, Croatia
}

\begin{abstract}
This paper evaluates the currents induced in the human body when exposed to extremely low frequency $(60 \mathrm{~Hz})$ electric fields by numerical modelling with boundary elements. The exposure is driven by the voltage of a rectangular panel representing the control keyboard of a power substation room. The main focus is to study the variations of the current density in the human body when located at different distances from the panel. In addition, the sensitivity of the results for the current density when considering the human body with and without its internal organs is estimated. The numerical approach is based on the three dimensional boundary element method (BEM) with the kernel of Laplace equation, in which the human body model considered is a simplified representation which allows parametric modifications of its geometry and physical properties.

Keywords: extremely low frequency, human exposure, boundary elements, power substations.
\end{abstract}

\section{Introduction}

Substation transformers are designed for a wide range of commercial, industrial, and utility applications. The usual activities of a power substation operator involve touching of control units, connecting and disconnecting switches as well as other grounded metallic objects exposed to high electrical fields. As a result, when a person is either in the proximity of or in contact with conductive surfaces at different potentials, induced and contact currents may flow throughout the different tissues. Pre contact spark discharge currents may also occur. There are well defined symptoms of acute exposure. At low frequencies, induced currents can cause effects on 
the function of the central nervous system. These well established acute effects will only occur as a result of intense exposure.

The exposure limit values on current density provided by the European directive 2004/40/EC on minimum health and safety requirements in the frequency range between $1 \mathrm{~Hz}$ and $10 \mathrm{MHz}$ are based on established adverse effects on the central nervous system. The limit on current density is intended to protect against acute exposure effects on central nervous system tissues in the head and trunk of the body. Such acute effects are essentially instantaneous. Due to the variation of electrical properties along different tissues of the body, the limit on current densities is prescribed over a cross section of $1 \mathrm{~cm}^{2}$ perpendicular to the current direction. The limit on current density is also provided as a basic restriction by the International Commision of Non-Ionizing Radiation Protection (ICNIRP) [1] and is limited $10 \mathrm{~mA} / \mathrm{m}^{2}$ across a $1 \mathrm{~cm}^{2}$ along head and trunk for workers and to $2 m A / m^{2}$ for general public. A particularly important exposure scenario to study is when the operator in the power substation room rises his arm in order to touch a keyboard at a certain voltage $V_{0}$. Considering the worst case scenario of feet in contact with earth, the extended arm, which can be seen as a thin long aspect ratio highly conductive object, will facilitate the development of non negligible currents throughout the body. Depending on the voltage level, the frequency and duration of the exposure, these currents inside the body may induce either short or long term adverse health conditions. Some epidemiological studies link increased rates of certain cancer, specially childhood leukemia, to occupations in which exposure to magnetic or electric fields is greater than average [2,3]. Hence, exposure guidelines and recommendations play a key role in this regard and may need to be revised. Numerical modelling represents an important tool in order to evaluate induced currents and electric fields, which are necessary inputs for further biological and medical studies at ELF fields. Induced currents in the body are highly dependent on the external geometrical conditions, especially when the body is grounded [4]. This paper deals with a realistic parametric model of the grounded human body standing inside a power substation room, in order to estimate the current density along the body and how it varies as the person gets closer to the electrified panel, which represents the driver of the problem. In addition, the influence of the different shapes and conductivities of some selected organs on the numerical results obtained is estimated.

The problem is described by Maxwell equations, which in the ELF approximation, reduce to the Laplace equation version of the conservation of charge. Hence, at ELF the current density $\mathbf{j}$ produced inside the body is obtained by solving the corresponding Laplace equation for the scalar potential $\varphi$. The solution approach is based on the boundary integral representation of the Laplace equation. Boundary element methods (BEM) [5] have an attractive advantage over other numerical methods, since they tend to avoid volume meshes and also their formulation is based on the fundamental solution of the leading operator for the governing equation, therefore being more accurate than standard Finite Element or Finite Difference methods. 


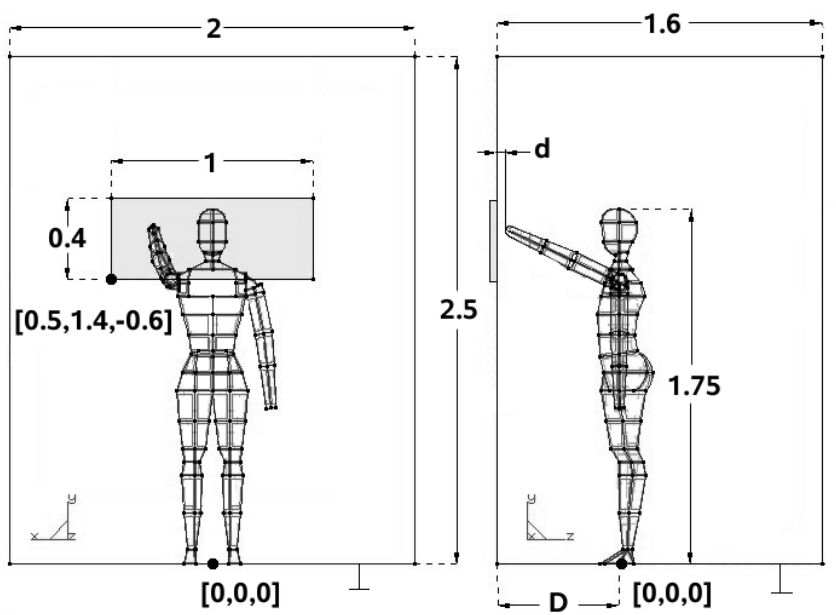

Figure 1: Main dimensions of the conceptual model representing a human body inside a substation room, extending his arm towards an electrified rectangular panel.

\section{Physical model}

The physical model under study contemplates a grounded, isolated human being with an arm pointing to a keyboard at a fixed potential and the air in its near environment. such as the case shown in Figure 1.

\subsection{Governing equations}

At low frequencies, the electric field $\mathbf{E}$ and magnetic field $\mathbf{H}$ are decoupled. So, directly from Maxwell's equations [6], under these assumptions, and considering that the conductivity $\sigma$, and the permittivity $\epsilon$ are constants within the considered region it is found that the electric scalar potential $\varphi$ obeys the following Laplace equation for the air and the human body:

$$
\nabla \cdot[(\sigma+i \omega \epsilon) \nabla \varphi]=0
$$

where $\omega$ is the frequency of the incident field, and $\epsilon$ is the permittivity.

\subsection{Interface matching conditions}

The conductivity and permittivity are both considered constant within each subdomain of the body. Considering the interface between two regions of different properties, the unit vector $\widehat{\mathbf{n}}$ is the normal of the surface diving the two media. 
Table 1: Selected tissue conductivities in $S / m$ from different sets of data for $60 H z$.

\begin{tabular}{lcc}
\hline Tissue Type & $\sigma_{\mathbf{L}}$ & $\sigma_{\mathbf{G}}$ \\
\hline Muscle & 0.50 & 0.35 \\
Heart & 0.11 & 0.10 \\
Brain & 0.12 & 0.06 \\
\hline
\end{tabular}

Prescribing the continuity of fluxes along the interface, leads to the following condition where $\partial_{n}:=\frac{\partial}{\partial n}$ and the superscripts 1 and 2 indicate the two media.

$$
\left[(\sigma+i \omega \epsilon) \partial_{n} \varphi\right]^{(1)}=\left[(\sigma+i \omega \epsilon) \partial_{n} \varphi\right]^{(2)}
$$

In general, $\varphi$ is regarded as a complex potential $\varphi=\varphi_{R}+i \varphi_{I}$, so (2) can be split into two equations. At ELF, conducting properties are dominant for human tissue, i.e. $\sigma^{(B I O)} \gg \omega \epsilon^{(B I O)}$ for the different biological tissues and the conductivity of the air is negligible [7]. Under the previous assumptions, equations (2) can be decoupled as presented below. First, it is possible to assign any arbitrary value for the phase of the potential in one of the media. Therefore, for the incident field, $\left[\varphi_{I}\right]^{(A I R)}$ can equals zero, resulting in the following expression for the interface between air and biological tissue:

$$
\left[\sigma \partial_{n} \varphi_{R}\right]^{(B I O)}=0 ; \quad\left[\sigma \partial_{n} \varphi_{I}\right]^{(B I O)}=\left[\omega \epsilon \partial_{n} \varphi_{R}\right]^{(A I R)}
$$

On the other hand, for interfaces mediating two regions of biological tissue ${ }^{(B I O 1)}$ and ${ }^{(B I O 2)}$, the following relations can be derived:

$$
\left[\sigma \partial_{n} \varphi_{R}\right]^{(B I O 1)}=\left[\sigma \partial_{n} \varphi_{R}\right]^{(B I O 2)} ; \quad\left[\sigma \partial_{n} \varphi_{I}\right]^{(B I O 1)}=\left[\sigma \partial_{n} \phi_{I}\right]^{(B I O 2)}
$$

\subsection{Human body model}

When dealing with human body modelling the major difficulties appearing are related to the material properties, as well as to geometrical aspects. At ELF frequencies biological tissues behave as good conductors with conductivity values of the order of $0.5 \mathrm{~S} / \mathrm{m}$; and the air represents a perfect dielectric [8,9].

In this paper the human body model considered is a simplified parametric representation in which the whole body is regarded as a saline fluid with conductivity $\sigma=0.5 \mathrm{~S} / \mathrm{m}$. Two different conceptual models for the human body are considered, namely $\mathrm{H}$ and $\mathrm{HO}$. $\mathrm{H}$ model is an homogeneous representation of the human body, while the HO model takes into account the presence of some relevant tissues like brain and heart. All organs are treated as conductors embedded in the saline fluid. Two sets of conductivity values have been considered for the organs. 


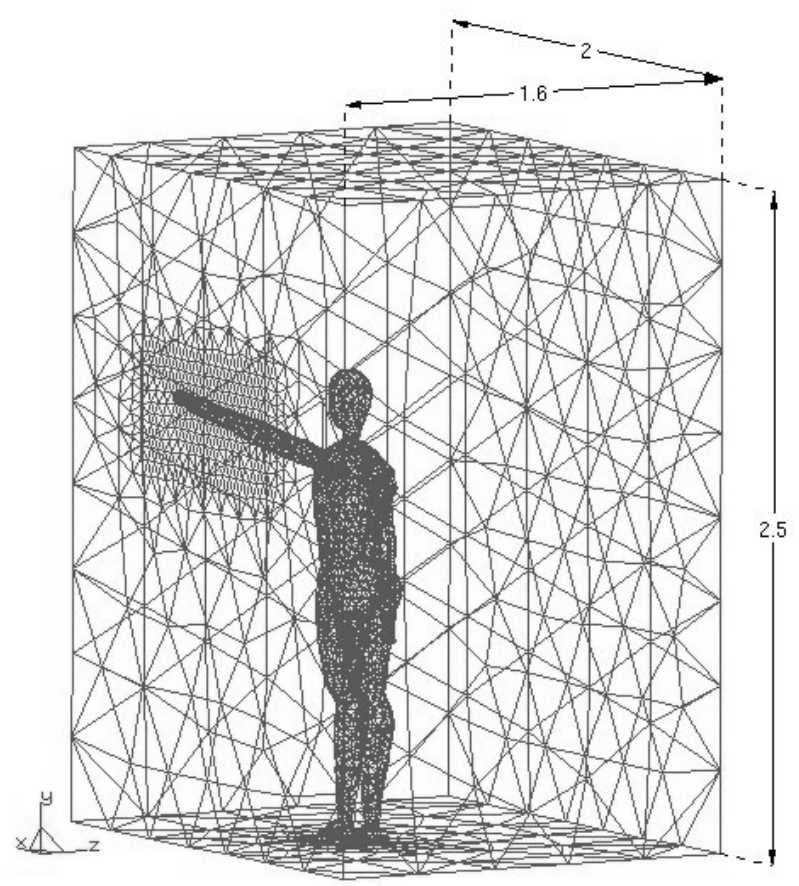

Figure 2: Boundary Element Mesh adopted for the model (5038 linear triangular elements).

The first set, $\sigma_{L}$, has been obtained from literature $[8,10,11,12]$. The second set, $\sigma_{G}$, from the measurements of Gabriel et al. $[13,14]$. The conductivities of the organs are relatively lower than its surrounding media. They are summarised in Table 2.3. The preprocessing and the geometrical introduction represented a major problem which has been sorted out by adapting a customizable geometry modeller, pre-processor and mesh generator [15]. The meshes generated have a number of elements ranging between 3,000 to 5,600 .

\section{Boundary Element Method}

The numerical implementation of the BEM requires the discretisation of the domain boundary into elements, and this represents one of the most powerful advantages of the method, since there is no need to discretise in volume. According to equation (1) the integral formulation of the governing differential equation evaluated over each subdomain $\omega_{[J]}$ represented by a particular tissue $[J]$, reduces to the 
following expression as has been described in [4].

$$
c_{i} \varphi_{i}+\int_{\Gamma_{(J)}} \frac{\partial g}{\partial n} \varphi d \Gamma_{[J]}-\int_{\Gamma} g \frac{\partial \varphi}{\partial n} d \Gamma_{[J]}+\int_{\Omega} \rho u^{*} d \Omega=0
$$

where $\Gamma_{(J)}=\Gamma_{1}+\Gamma_{2}$ (the boundary of $\Omega_{(J)}$ ), $n$ is the normal to the boundary $\Gamma_{(J)}$. And the weight function $g$ is chosen to be the free space Green function of the Laplace operator. Equation (5) represents the integral formulation of (1), and is the starting point of the BEM code that has been implemented.

\section{Results and discussion}

The conceptual model of the case considered consists of a realistic human body inside a power substation room. The room is modelled as a $2.5 \mathrm{~m}$ high, $1.6 \mathrm{~m}$ wide and $2 \mathrm{~m}$ long closed parallelepiped surrounding the human body as shown in Fig. 1. The $1.75 \mathrm{~m}$ tall human being is facing a hypothetical control panel $1 \mathrm{~m}$ wide and $0.4 \mathrm{~m}$ high, at the level of the head. The lower right (right from the human body point of view) corner of the model is located at coordinates $(x, y, z)=$ $(0.5,1.4,-0.6 \mathrm{~m})$, when taking into account the origin at the intersection between the vertical longitudinal axis of the human body and the ground floor, indicated as $(0,0,0)$ in Fig. 1 .

Two different conditions will be evaluated on the base of this model, namely $\mathrm{A}$ and $\mathrm{B}$. The former considers a gap $d=0.016 \mathrm{~m}$ between the person's hand and the panel, while the latter is shifted $10 \mathrm{~cm}$ further away from the panel, i.e. $d=0.116 \mathrm{~m}$. The gap is considered as a dielectric material (dry air). The boundary conditions applied are as follows. The panel has fixed potential $V_{0}=400 \mathrm{~V}$ while the floor is kept grounded and all other surfaces of the room are considered with Neumann adiabatic type conditions (i.e. $\nabla \varphi \cdot \hat{n}=0$ ). Because of the linearity of the problem the results shown in this calculation can be scaled to any other value of applied voltage $V_{0}$. Figure 2 shows one of the meshed model, with 5038 flat linear triangular elements and 2506 nodes.

Next, the induced current in the different conceptual models of the body at the two different scenarios and for both conductivity distributions is analysed for a total of 8 data sets. Figure 3 shows the absolute value of the current density $|\mathbf{j}|=$ $\sqrt{j_{x}^{2}+j_{y}^{2}+j_{z}^{2}}$ along the vertical $y$ direction. The observation lines goes along the centroid of the crotch, torso neck and head.

The figure shows a comparison between the $\mathrm{HO}$ and $\mathrm{H}$ models at the smallest distance between hand and panel for both conductivity distributions. It can be observed that the consideration of rough approximations of the internal organs introduce slight modifications of the general behaviour predicted by the homogeneous model $(\mathrm{H})$. The profile is mostly dominated by the external shape and exposure conditions and a second order correction appears when keeping into account the organs. The presence of the heart introduces a fluctuation of nearly $0.2 \times 10^{-2} \mathrm{~mA} / \mathrm{m}^{2}$ in the current density observed outside it. The consideration of the brain in the model introduces a similar deviation in the current density. In 


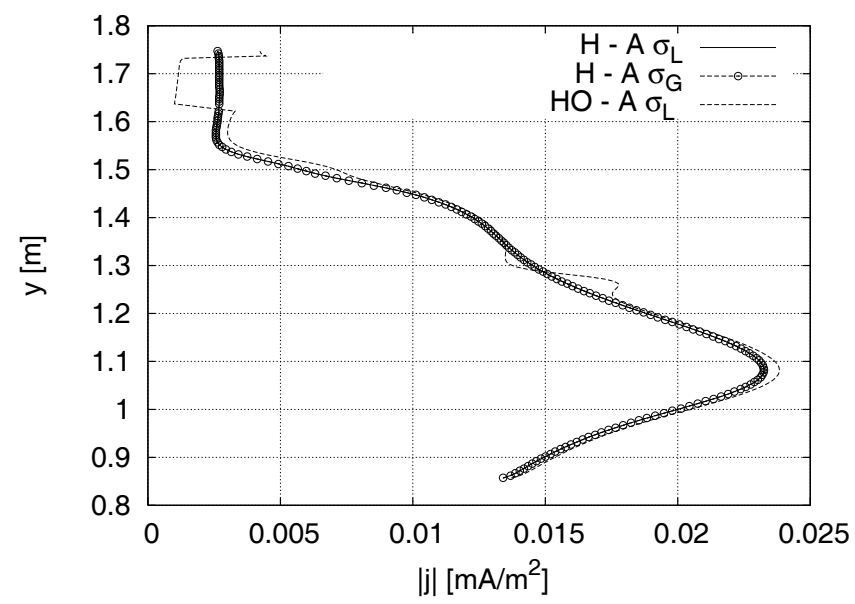

Figure 3: Absolute Value of the current density along the torso for the $\mathrm{H}$ and $\mathrm{HO}$ models in the exposure scenario A for both conductivity distributions $\sigma_{L}$ and $\sigma_{G}$.

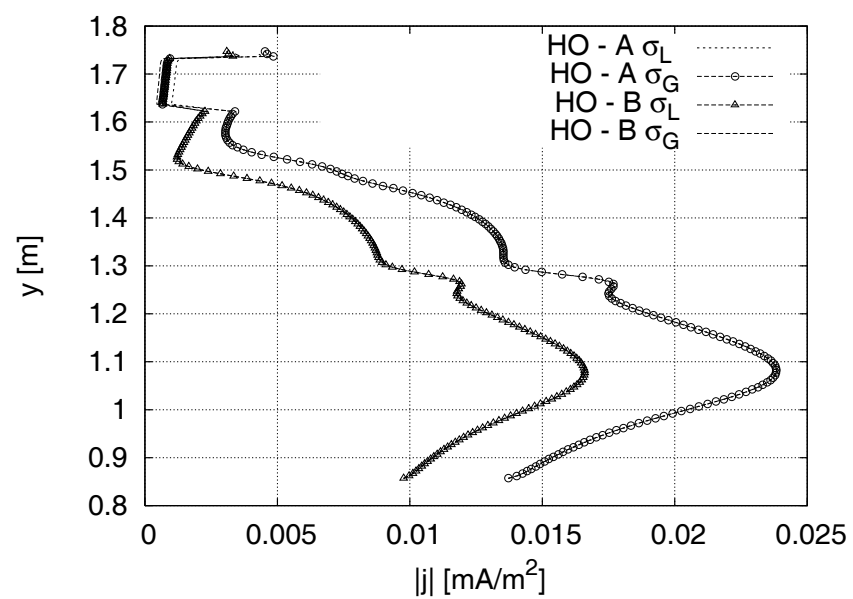

Figure 4: Absolute Value of the current density along the torso for the HO model in the two different exposures scenarios A and B for both conductivity distributions $\sigma_{L}$ and $\sigma_{G}$.

addition, the maximum peak of approximately $2.4 \times 10^{-3} \mathrm{~mA} / \mathrm{m}^{2}$ is located near the waist, invariable where the cross section is slightly reduced. We reason that the effect of a local depression in the sectional area normally exposed to the driving field is the most important factor that determines the profile of induced currents inside the body. 


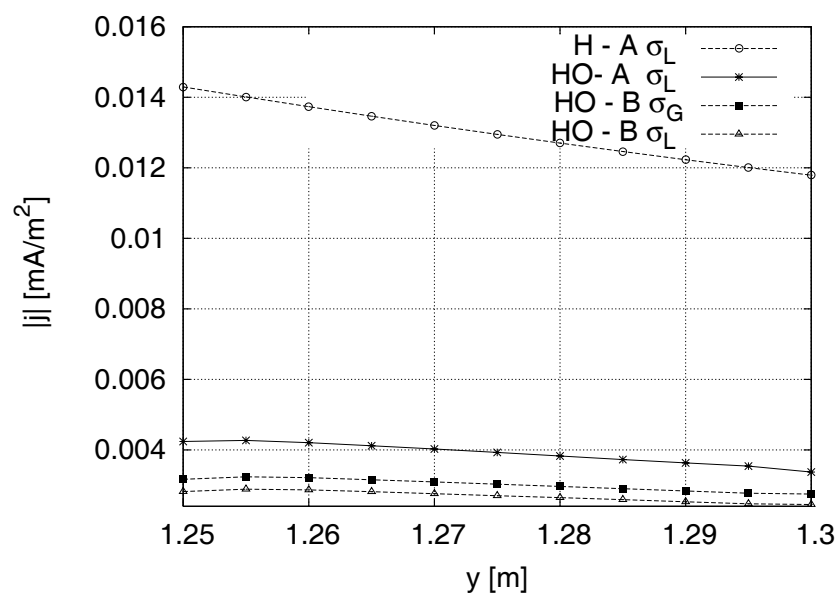

Figure 5: Absolute value of current density along the heart at the two exposure conditions and both conductivity distribution.

Figure 4 shows the absolute value of the current density along the vertical $y$ direction for the $\mathrm{HO}$ models at two different exposure scenarios for both conductivity distributions. As expected, current densities in scenario A are nearly $50 \%$ greater than in B. Both conductivity distributions show nearly the same values of current density. Figure 5 shows the absolute value of the current density inside the heart along the vertical $y$ direction for scenarios $\mathrm{A}$ and $\mathrm{B}$, and both conductivity distributions. The current density in the heart decays roughly $1 \mu \mathrm{A} / \mathrm{m}^{2}$ when shifted $10 \mathrm{~cm}$ away from the panel. The maximum absolute value of current density found in the heart is: $3.2 \mu \mathrm{A} / \mathrm{m}^{2}$ and $4.4 \mu \mathrm{A} / \mathrm{m}^{2}$ for case scenario $\mathrm{A}$ and $\mathrm{B}$, respectively. The values found are way below the recommended guidelines at ELF [1]. As expected, the current density induced under exposure scenario B is lower than under scenario A. The values obtained for both conductivity distributions are similar for all the models considering organs, and \% lower than the correspondent values for the homogeneous model H. Finally, Figure 6 shows a 3D view of the current density distribution for the $\mathrm{H}$ model in scenario $\mathrm{A}$. The arrows have constant length (i.e they are not in scale with the values of current density, but show the main flow direction). The mostly exposed region of the body is the arm extended towards the panel, while the other one is basically unaffected.

\section{Conclusion}

An accurate three dimensional BEM approach based on the kernel of Laplace equation has been developed and successfully applied for the study of the currents induced in the human body when exposed to an extremely low frequency $(60 \mathrm{~Hz})$ 


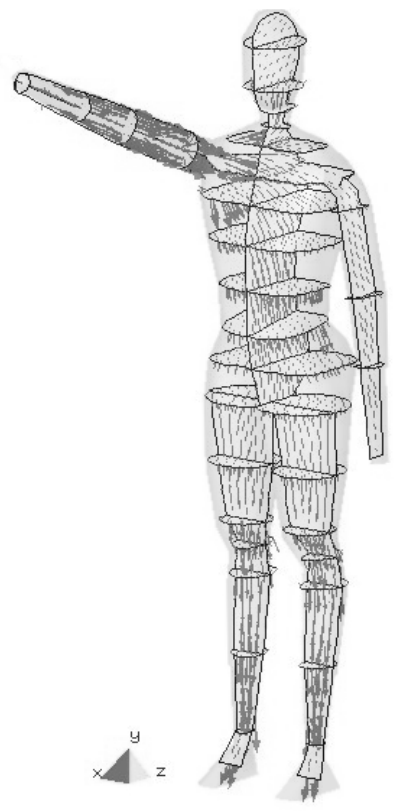

Figure 6: Three dimensional current density distribution inside the homogeneous model of the human body.

electric field. The case study analysed consists of the human body inside a power substation room, with his arm extended towards a hypothetical electrified panel. The problem is driven by the voltage $V_{0}$ of this panel, regarded as a control keyboard representing the control keyboard, whose voltage is assigned as a boundary condition.

The current density along the vertical axis of the body when located at two different distances from the panel has been accurately calculated, for the case of grounded body and non-contacting conditions between hand and panel (i.e, the gap between hand and panel is air, in dry conditions).

The study reveals that the maximum peak of current occurs near the waist, around the region of reduced cross section. When the hand approximates the keyboard tough without touching it, considering a $1.6 \mathrm{~cm}$ gap of dry air in between, the maximum current density observed in the waist is approximately $2.4 \times 10^{-2} \mathrm{~mA} / \mathrm{m}^{2}$, assuming a panel voltage $V_{0}=400 \mathrm{~V}$. Then, when shifted $10 \mathrm{~cm}$ apart, the maximum value decreases to nearly $1.6 \times 10^{-2} \mathrm{~mA} / \mathrm{m}^{2}$.

The calculation has been repeated for a homogeneous human body with $\sigma \sim$ $0.5 \mathrm{~S} / \mathrm{m}$ and a heterogeneous one, which includes nearly ellipsoidal models of heart and brain with reduced conductivity $(\sigma \sim 0.11 \mathrm{~S} / \mathrm{m})$. Two sets of conductivity values has been assign to the different tissues. The first set has been extracted from values reported in the literature, while the second set was based on the mea- 
surements performed by Gabriel et al, The overall result for the 8 data sets calculated is nearly the same, except that the induced current inside those tissues is significantly modified. In all cases the location and magnitude of the maximum peak of current density is mostly dominated by the external shape of the body, rather than by the distribution of internal non-homogeneous inclusions.

\section{References}

[1] ICNIRP. Guidelines for limiting exposure to time-varying electric, magnetic, and electromagnetic fields (up to $300 \mathrm{ghz}$ ). Health Physics. 74(4):494-522, 1998.

[2] M. J. Crumpton and A. R. Collins. Are environmental electromagnetic fields genotoxic? DNA Repair 3(10):1385-87, 2004.

[3] S.Ivancsits, E.Diem, H. R. A. Pilger, and O. Jahn. Induction of dna strand breaks by intermittent exposure to extremely-low-frequency electromagnetic fields in human diploid fibroblasts. JCMOSS 519(1-2):1-13, 2002.

[4] C. Gonzalez, A. Peratta, and D. Poljak. Boundary element modeling of the realistic human body exposed to extremely low frequency (elf) power distributions lines using the boundary element method. IEEE Trans. on Electromagnetic Compatibility 49(1):153-162, 2007.

[5] C. Brebbia, J. C. Telles, and L. C. Wrobel. Boundary Elements Techniques. Springer-Verlag, Berlin, Heidelberg New York and Tokio, 1984.

[6] J. Jackson. Classical Electrodynamics. J.Willey \& Sons, New York, 1975.

[7] N.S.Stoykov. Frequency and time domanin fem models fo emg: Capacitive effects and aspects of dispersion. IEEE Trans.on Biom. Eng. 49(8), 2002.

[8] O. Gandhi. Some numerical methods for dosimetry: Extremely low frequencies to microwave frequencies. Radio Sci. 30(1):161-177, 1995.

[9] R.W.P.King. A review of analytically determined electric fields and currents induced in the human body when exposed to 50-60 hz electromagnetics fields. IEEE Trans.on Antennas and Propagation 52(5):1186-1192, 1998.

[10] A. Chiba, K.Isaka, Y.Yokoi, M.Nagata, M. Kitagav, and T. Matsuo. Application of finite element method to analysis of induced current densities inside human model exposed to $60 \mathrm{hz}$ electric field. IEEE Trans. Power Apparatus and Systems 103(7):1895-1901, 1984.

[11] O. Gandhi and J. Chen. Numerical dosimetry at power line frequencies using anatomically based models. Bioelectromagnetics Suppl. 1:43-60, 1992.

[12] R. King and S. Sandler. Electric fields and currents induced in organs of the human body when exposed to ELF and VLF electromagnetic fields. Radio Sci 31:1153-1167, 1996.

[13] C. Gabriel, S. Gabriel, and E. Corthout. The dielectric properties of biological tissues: I. literature survey. Phys. Med. Biol. 41:2231-49, 1996.

[14] S.Gabriel, R. Lau, and C. Gabriel. The dielectric properties of biological tissues: Ii. measurements in the frequency range $10 \mathrm{hz}$ to $20 \mathrm{ghz}$ phys. Phys. Med. Biol. 41:2251-69, 1996.

[15] GID resources. Website, http://gid.cimne.upc.es. 